\title{
INTERAÇÃO DE IMUNOGLOBULINA G HUMANA EM ADSORVENTES DE QUITOSANA/ALGINATO: AVALIAÇÃO DE ISOTERMAS DE ADSORÇÃO E PARÂMETROS TERMODINÂNICOS
}

\author{
N. M. Millan ${ }^{1}$, A. C. M. Pássaro ${ }^{1}$, T. M. Mozetic ${ }^{1}$, I. J. Silva Jr² e I. T. L. Bresolin ${ }^{1}$ \\ ${ }^{1}$ Universidade Federal de São Paulo, Departamento de Engenharia Química \\ ${ }^{2}$ Universidade Federal do Ceará, Centro de Tecnologia, Departamento de Engenharia \\ Química
}

\begin{abstract}
RESUMO - Imunoglobulinas humanas, com destaque à IgG, são anticorpos com ampla aplicação terapêutica no tratamento de doenças do sistema imunológico, o que justifica a sua necessidade de alta pureza, normalmente atingida com métodos cromatográficos. Este trabalho estudou a capacidade de microesferas de quitosana/alginato sem ligantes imobilizados de adsorver IgG humana, em diferentes temperaturas de $4^{\circ} \mathrm{C}, 15^{\circ} \mathrm{C}, 25^{\circ} \mathrm{C}$ e $37^{\circ} \mathrm{C}$ com tampões HEPES (pH 6,8) e MES (pH 6,5). Observou-se que a matriz possui afinidade média e que o tampão HEPES proporcionou as maiores capacidades de adsorção, em especial em $4^{\circ} \mathrm{C}$, e que ocorre cooperatividade positiva no sistema, que se ajusta melhor ao modelo de Langmuir-Freundlich. Os parâmetros termodinâmicos foram determinados e revelaram um processo de adsorção espontâneo e a possibilidade de interações hidrofóbicas e adsorção em multicamada.
\end{abstract}

\section{INTRODUÇÃO}

Imunoglobulinas humanas são glicoproteínas que atuam no reconhecimento de antígenos. Dentre estas, destaca-se a imunoglobulina da classe G (IgG) por sua ampla aplicação em fins terapêuticos e também diagnósticos, como no tratamento das imunodeficiências primárias. Essas somam mais de 150 doenças e, em sua maioria, resultam em maior suscetibilidade a infecções, doenças autoimunes e neoplasias. A imunoglobulina humana como terapia de reposição de anticorpos tem sido recomendada nesses casos, a fim de reduzir o risco de infecções e suas sequelas (Carvalho et al., 2010).

Essas aplicações requerem IgG com alto grau de pureza, dado que a presença de outros tipos de proteínas pode comprometer a eficácia do processo (Prasanna e Vijayalakshmi, 2010). Atualmente, os processos tradicionais de purificação de imunoglobulinas (como precipitação por adição de sais ou variações de temperatura e $\mathrm{pH}$ ) vêm sendo substituídos por métodos mais seletivos baseados em cromatografia de adsorção (Lowe et al., 2001). A cromatografia de afinidade tem sido cada vez mais abordada, fazendo-se uso dos mais variados ligantes (proteínas A e G, corantes, aminoácidos, agentes tiofílicos e íons metálicos)

No entanto, dependendo dos grupamentos disponíveis na superfície do próprio suporte cromatográfico, pode-se também pensar em sua utilização sem nenhum ligante imobilizado. Neste sentido, o presente trabalho buscou avaliar a capacidade de adsorção de IgG humana 
em microesferas de quitosana/alginato epoxiladas por meio da obtenção de isotermas de adsorção (Langmuir e Langmuir-Freundlich). Também foram levantados os parâmetros termodinâmicos da adsorção: energia livre de Gibbs $\left(\Delta \mathrm{G}^{0}\right)$, a entalpia $\left(\Delta \mathrm{H}^{0}\right)$ e a entropia $\left(\Delta \mathrm{S}^{0}\right)$.

\section{MATERIAIS E MÉTODOS}

\subsection{Materiais}

2.1.1. Reagentes: HEPES (ácido N-2-hidroxietilpiperazino-N'-2-etanosulfônico) e MES (ácido morfolinoetanosulfônico) foram adquiridos da Sigma (EUA). A IgG humana utilizada foi o produto comercial Beriglobina ${ }^{\circledR} 160 \mathrm{mg} / \mathrm{mL}$ (CSL Behring, Alemanha). O preparo dos tampões foi feito com água destilada. As microesferas de quitosana/alginato epoxiladas foram sintetizadas e fornecidas pelo Grupo de Pesquisa em Separações por Adsorção (GPSA) do Departamento de Engenharia Química da Universidade Federal do Ceará.

\subsection{Métodos}

2.2.1. Ensaios em batelada: Os experimentos de obtenção de isotermas de adsorção foram realizados em duplicata. Adicionou-se, a tubos eppendorf de $1,5 \mathrm{~mL}, 5 \mathrm{mg}$ do adsorvente e $1 \mathrm{~mL}$ de solução de proteína em tampão (concentração variando de 0,5 a 10,0 $\mathrm{mg} / \mathrm{mL}$ ). Estes tubos foram submetidos a uma agitação de 3 horas em um agitador orbital com incubadora a $120 \mathrm{rpm}$ (Infors HT, UK) e, em seguida, o sobrenadante foi quantificado pelo método de Bradford (1976) a $595 \mathrm{~nm}$ em um espectrofotômetro UV-Vis (Evolution 60S, Thermo Scientific, EUA). Foram realizados experimentos em quatro temperaturas $\left(4^{\circ} \mathrm{C}\right.$, $15^{\circ} \mathrm{C}, 25^{\circ} \mathrm{C}$ e $\left.37^{\circ} \mathrm{C}\right)$ em meio a dois tampões diferentes: HEPES ( $\left.\mathrm{pH} 6,8\right)$ e MES (pH 6,5).

2.2.2. Determinação das isotermas de adsorção: Cada ponto da isoterma foi obtido com a média das duplicatas e a capacidade de adsorção foi obtida com um balanço de massa (Equação 1). Os gráficos de $\mathrm{q}^{*}$ em função de $\mathrm{C}^{*}$ foram construídos e os parâmetros dos modelos de Langmuir (Equação 2) e Langmuir-Freundlich (Equação 3)s foram ajustados, por regressão não linear usando o software OriginPro $8^{\circledR}$ (OriginLab Corporation, EUA)..

$$
\begin{aligned}
& \mathrm{q}^{*}=\frac{\mathrm{V}_{\mathrm{sol}}\left(\mathrm{C}_{0}-C^{*}\right)}{\mathrm{m}_{\mathrm{ads}}} \\
& \mathrm{q}^{*}=\frac{\mathrm{q}_{\mathrm{m}} \mathrm{C}^{*}}{\mathrm{~K}_{\mathrm{d}}+\mathrm{C}^{*}} \\
& \mathrm{q}^{*}=\frac{\mathrm{q}_{\mathrm{m}}\left(\mathrm{C}^{*}\right)^{n}}{\mathrm{~K}_{\mathrm{d}(\mathrm{LF})}+\left(C^{*}\right)^{n}}
\end{aligned}
$$

Em que $q^{*}$ é a quantidade de proteína adsorvida por grama de adsorvente $(\mathrm{mg} / \mathrm{g}), \mathrm{C}^{*}$ é a concentração de proteína na fase líquida $(\mathrm{mg} / \mathrm{mL})$ em equilíbrio com $q^{*}, \mathrm{C}_{\mathrm{O}}$ é a concentração inicial de proteína na fase líquida $(\mathrm{mg} / \mathrm{mL})$, mads é a massa de adsorvente $(\mathrm{g})$ e Vsol é o volume da solução $(\mathrm{mL})$ em contato com o adsorvente. A variável $q_{m}$ é a capacidade máxima de proteínas adsorvidas (em $\mathrm{mg} / \mathrm{g}), K_{D}$ e $K_{D(L F)}$ são as constantes de dissociação e de dissociação aparente $(\mathrm{mol} / \mathrm{L})$, respectivamente, e $n$ é a constante de Langmuir-Freundlich. 
2.2.3 Determinação dos parâmetros termodinâmicos: A partir das constantes de dissociação obtidas pelo ajuste dos parâmetros ao modelo de Langmuir e com o auxílio do software Origin $^{\circledR}$, foram determinadas as propriedades termodinâmicas da adsorção, tais como $\Delta \mathrm{G}^{0}, \Delta \mathrm{H}^{0}$ e $\Delta \mathrm{S}^{0}$. $A \Delta \mathrm{G}^{0}$ foi determinada pela definição da energia livre de Gibbs (Equação 4) ao se considerar $\Delta \mathrm{G}$ nulo no equilíbrio. A constante $\mathrm{K}_{\mathrm{d}}$ se relaciona com a temperatura por meio da relação de Van’t Hoff na forma integrada e linearizada (Equação 5). Já o valor de $\Delta \mathrm{S}^{0}$ foi encontrado pela relação de Gibbs-Helmholtz (Equação 6).

$$
\begin{aligned}
& \Delta G=\Delta G^{0}-R T \ln K_{d} \\
& \ln K_{d}=\frac{\Delta H^{0}}{R T}+J \\
& \Delta G^{0}=\Delta H^{0}-T \Delta S^{0}
\end{aligned}
$$

Em que R é a constante universal dos gases e J é a constante de integração.

\section{RESULTADOS E DISCUSSÃO}

\subsection{Isotermas de Adsorção}

Os parâmetros obtidos pelo ajuste não linear dos dados de adsorção de IgG no adsorvente utilizando-se o tampão HEPES $(25$ mmol/L e pH 6,8) estão na Tabela 1.

Tabela 1 - Parâmetros obtidos a partir do ajuste não linear dos modelos de Langmuir e Langmuir-Freundlich aos dados de adsorção (HEPES 25 mmol/L, pH 6,8).

\begin{tabular}{|c|c|c|c|c|c|c|c|}
\hline \multirow{2}{*}{$\begin{array}{c}\text { Temperatura } \\
\left({ }^{\circ} \mathrm{C}\right)\end{array}$} & \multicolumn{5}{|c|}{ Langmuir } & \multicolumn{3}{c|}{ Langmuir-Freundlich } \\
\cline { 2 - 8 } & $\begin{array}{c}\mathrm{Q}_{\mathrm{m}} \\
(\mathrm{mg} / \mathrm{g} \text { seca })\end{array}$ & $\begin{array}{c}\mathrm{K}_{\mathrm{d}} \\
(\mathrm{mol} / \mathrm{L})\end{array}$ & $\mathrm{R}^{2}$ & $\begin{array}{c}\mathrm{Q}_{\mathrm{m}} \\
(\mathrm{mg} / \mathrm{g} \mathrm{seca})\end{array}$ & $\begin{array}{c}\mathrm{K}_{\mathrm{d}(\mathrm{LF})} \\
(\mathrm{mol} / \mathrm{L})\end{array}$ & $\mathrm{n}$ & $\mathrm{R}^{2}$ \\
\hline \hline 4 & $\begin{array}{c}919,66 \pm \\
418,58\end{array}$ & $\begin{array}{c}(2,93 \pm \\
2,13) \times 10^{-5}\end{array}$ & 0,920 & $\begin{array}{c}455,21 \pm \\
76,50\end{array}$ & $\begin{array}{c}(1,54 \pm \\
0,59) \times 10^{-5}\end{array}$ & $2,37 \pm 0,89$ & 0,953 \\
\hline 15 & $\begin{array}{c}285,40 \pm \\
26,02\end{array}$ & $\begin{array}{c}(6,61 \pm \\
1,91) \times 10^{-6}\end{array}$ & 0,962 & $\begin{array}{c}226,01 \pm \\
6,95\end{array}$ & $\begin{array}{c}(2,62 \pm \\
0,61) \times 10^{-7}\end{array}$ & $2,16 \pm 0,30$ & 0,993 \\
\hline 25 & $\begin{array}{c}340,13 \pm \\
24,63\end{array}$ & $\begin{array}{c}(9,05 \pm \\
2,17) \times 10^{-6}\end{array}$ & 0,962 & $\begin{array}{c}285,78 \pm \\
14,01\end{array}$ & $\begin{array}{c}(6,45 \pm \\
1,28) \times 10^{-6}\end{array}$ & $1,67 \pm 0,27$ & 0,984 \\
\hline 37 & $387,64 \pm$ & $\begin{array}{c}(2,10 \pm \\
0,68) \times 10^{-6}\end{array}$ & 0,955 & $\begin{array}{c}284,15 \pm \\
22,23\end{array}$ & $\begin{array}{c}(1,76 \pm \\
0,41) \times 10^{-5}\end{array}$ & $1,86 \pm 0,41$ & 0,979 \\
\hline
\end{tabular}

Pelos coeficientes de correlação na Tabela 1, próximos de 1,0, percebe-se que os dois modelos se ajustaram bem. No entanto, o modelo de Langmuir-Freundlich teve um ajuste melhor, pois se aproxima mais de um sistema não ideal ao considerar a existência de sítios não uniformes e de heterogeneidade das interações entre proteína e adsorvente. (Bresolin et al., 2010) Percebe-se também que Langmuir-Freundlich apresentou $n>1$ no ajuste, indicando cooperatividade positiva: a adsorção de uma molécula de $\operatorname{IgG}$ favorece a adsorção de outra, possivelmente devido à característica heterogênea da adsorção. (Bresolin et al., 2010)

Em seu trabalho, Gondim et al. (2014) abordou ensaios em leito fixo de adsorção de IgG humana nas mesmas microesferas de quitosana/alginato utilizadas neste trabalho, porém 
com o corante Cibacron Blue F3GA imobilizado. Neste caso, obteve-se que, a uma temperatura de $25^{\circ} \mathrm{C}$ e tampão HEPES de mesma força iônica $(25 \mathrm{mmol} / \mathrm{L})$ e mesmo $\mathrm{pH}$ $(6,8)$, o modelo de Langmuir fornecia um valor de $Q_{m}$ de $93,3 \mathrm{mg} / \mathrm{g}$ seca e o de LangmuirFreundlich, $85,4 \mathrm{mg} / \mathrm{g}$ seca. Estes valores, quando comparados com aqueles que estão na Tabela 1 para $25^{\circ} \mathrm{C}$, se mostram inferiores nos dois modelos. Isto sugere que a matriz sem ligantes imobilizados possui, quando usada em ensaios de batelada, uma capacidade de adsorção maior que a matriz com Cibracron Blue F3G4 imobilizado em ensaios de leito fixo.

Também se infere da Tabela 1 que ambos os modelos forneceram o maior valor de $\mathrm{Q}_{\mathrm{m}} \mathrm{a}$ $4^{\circ} \mathrm{C}$. Com relação a $\mathrm{K}_{\mathrm{d}}$, o modelo de Langmuir mostrou uma maior afinidade entre a $\operatorname{IgG}$ e a matriz na em $37^{\circ} \mathrm{C}$, enquanto que para Langmuir-Freundlich isso ocorreu a $15^{\circ} \mathrm{C}$. Todos os dados de $\mathrm{K}_{\mathrm{d}}$ obtidos se mostraram condizentes com os valores típicos de sistemas de pseudobioafinidade (faixa de $10^{-2}$ a $10^{-7} \mathrm{~mol} / \mathrm{L}$ ), indicando um nível médio de afinidade (Bresolin et al., 2010).

Por sua vez, os parâmetros obtidos pelo ajuste não linear dos dados de adsorção em meio tampão MES (25 mmol/L e pH 6,5) estão na Tabela 2.

Tabela 2 - Parâmetros obtidos a partir do ajuste não linear dos modelos de Langmuir e Langmuir-Freundlich aos dados de adsorção (MES 25 mmol/L, pH 6,5).

\begin{tabular}{|c|c|c|c|c|c|c|c|}
\hline \multirow{2}{*}{$\begin{array}{c}\text { Temperatura } \\
\left({ }^{\circ} \mathrm{C}\right)\end{array}$} & \multicolumn{3}{|c|}{ Langmuir } & \multicolumn{3}{c|}{ Langmuir-Freundlich } \\
\cline { 2 - 8 } & $\begin{array}{c}\mathrm{Q}_{\mathrm{m}} \\
(\mathrm{mg} / \mathrm{g} \text { seca })\end{array}$ & $\begin{array}{c}\mathrm{K}_{\mathrm{d}} \\
(\mathrm{mol} / \mathrm{L})\end{array}$ & $\mathrm{R}^{2}$ & $\begin{array}{c}\mathrm{Q}_{\mathrm{m}} \\
(\mathrm{mg} / \mathrm{g} \text { seca })\end{array}$ & $\begin{array}{c}\mathrm{K}_{\mathrm{d}(\mathrm{LF})} \\
(\mathrm{mol} / \mathrm{L})\end{array}$ & $\mathrm{n}$ & $\mathrm{R}^{2}$ \\
\hline \hline 4 & $\begin{array}{c}430,73 \pm \\
69,72\end{array}$ & $\begin{array}{c}(1,48 \pm \\
0,58) \times 10^{-5}\end{array}$ & 0,936 & $\begin{array}{c}319,90 \pm \\
31,77\end{array}$ & $\begin{array}{c}(1,11 \pm \\
0,32) \times 10^{-5}\end{array}$ & $1,86 \pm 0,47$ & 0,967 \\
\hline 15 & $\begin{array}{c}154,87 \pm \\
12,13\end{array}$ & $\begin{array}{c}(6,84 \pm \\
1,80) \times 10^{-6}\end{array}$ & 0,969 & $\begin{array}{c}134,04 \pm \\
12,63\end{array}$ & $\begin{array}{c}(4,62 \pm \\
1,77) \times 10^{-6}\end{array}$ & $1,46 \pm 0,41$ & 0,976 \\
\hline 25 & $\begin{array}{c}215,68 \pm \\
20,60\end{array}$ & $\begin{array}{c}(8,59 \pm \\
2,50) \times 10^{-6}\end{array}$ & 0,960 & $\begin{array}{c}170,59 \pm \\
7,20\end{array}$ & $\begin{array}{c}(4,62 \pm \\
1,02) \times 10^{-6}\end{array}$ & $2,01 \pm 0,33$ & 0,989 \\
\hline 37 & $\begin{array}{c}362,14 \pm \\
34,65\end{array}$ & $\begin{array}{c}(1,94 \pm \\
0,42) \times 10^{-5}\end{array}$ & 0,979 & $\begin{array}{c}302,20 \pm \\
30,52\end{array}$ & $\begin{array}{c}(1,72 \pm \\
0,30) \times 10^{-5}\end{array}$ & $1,35 \pm 0,23$ & 0,987 \\
\hline
\end{tabular}

Assim como com o tampão HEPES, ambos os modelos tiveram coeficientes de correlação próximos de 1,0, com Langmuir-Freundlich apresentando o melhor ajuste. O maior valor de $\mathrm{Q}_{\mathrm{m}}$, nos dois modelos, se deu em $4^{\circ} \mathrm{C}$. Quanto à constante $\mathrm{K}_{\mathrm{d}}$, observou-se uma maior afinidade entre $\mathrm{IgG}$ e suporte em $15^{\circ} \mathrm{C}$ no modelo de Langmuir e em $15^{\circ} \mathrm{C}$ e $25^{\circ} \mathrm{C}$ (as constantes foram iguais) em Langmuir-Freundlich. Foi observado cooperatividade positiva novamente e que os valores de $K_{d}$ também se situam na faixa característica de sistemas de pseudobioafinidade, igualmente com afinidade média.

No trabalho de Gondim et al. (2014), não foi feito o ajuste para os dados de adsorção em meio a tampão MES. Porém, ao se investigar apenas a influência do $\mathrm{pH}$ em quatro tampões diferentes que incluíam MES $(\mathrm{pH}=6,7)$ e HEPES $(\mathrm{pH}=6,8)$ em ensaios batelada, verificou-se também que a adsorção em tampão HEPES possuía resultados maiores que a adsorção em tampão MES.

Na Figura 1, observam-se as isotermas obtidas, para os dois tampões, com o modelo de melhor ajuste, que foi Langmuir-Freundlich. 
Figura 1 - Isotermas de adsorção de IgG em matriz quitosana/alginato com tampão HEPES 25 mmol/L e pH 6,8: ajuste não linear dos parâmetros segundo o modelo de Langmuir (esquerda) e Langmuir-Freundlich (direita).
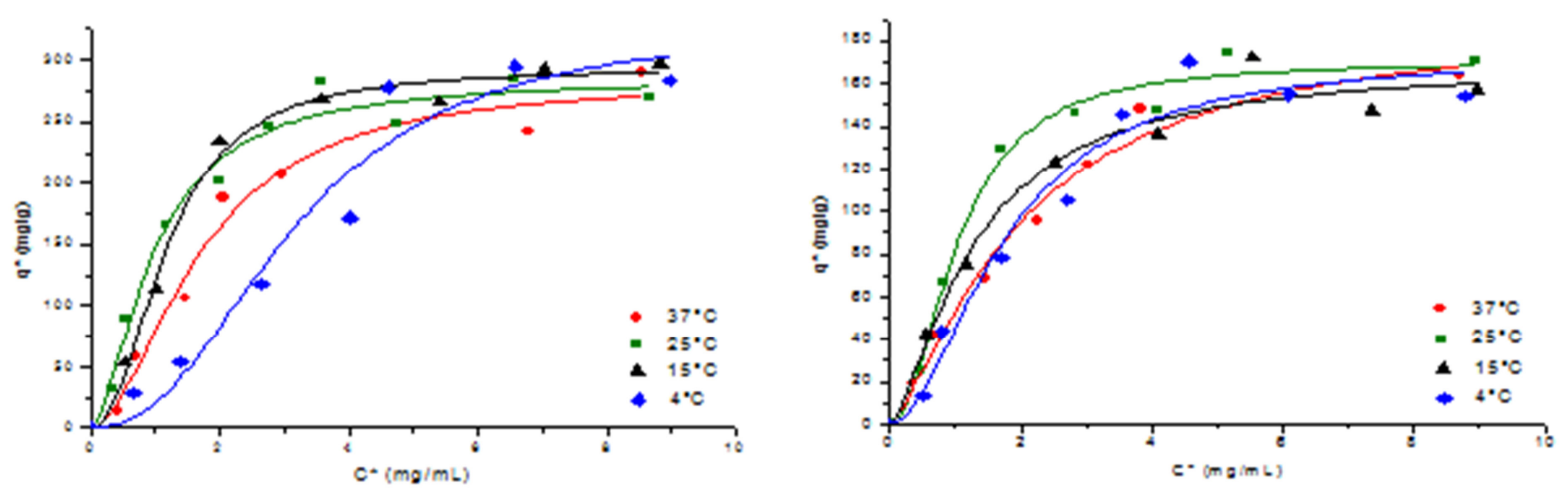

Pela Figura 1, observa-se que há concordância com o R² da Tabela 1 e da Tabela 2.

\subsection{Determinação dos Parâmetros Termodinâmicos}

Os valores de $\Delta \mathrm{G}^{\circ}$ e $\Delta \mathrm{S}^{\circ}$, calculados pela Equação 4 e pela Equação 6, respectivamente, e o valor de $\Delta \mathrm{H}^{\circ}$, encontrado pelo coeficiente angular da Equação 5, estão na Tabela 3.

Tabela 3 - Parâmetros termodinâmicos para a adsorção de IgG em matriz de quitosana/alginato com tampão HEPES $(25 \mathrm{mmol} / \mathrm{L}$ e pH 6,8) e MES $(25 \mathrm{mmol} / \mathrm{L} \mathrm{e} \mathrm{pH} \mathrm{6,5).}$

\begin{tabular}{|c|c|c|c|c|}
\hline Tampão & Temperatura $\left({ }^{\circ} \mathrm{C}\right)$ & $\Delta \mathrm{G}^{\circ}(\mathrm{kJ} / \mathrm{mol})$ & $\Delta \mathrm{H}^{\circ}(\mathrm{J} / \mathrm{mol})$ & $\Delta \mathrm{S}^{\circ}(\mathrm{J} / \mathrm{mol})$ \\
\hline \hline \multirow{4}{*}{ MES } & 4 & $-252,74$ & & \multirow{3}{*}{$+261,11$} \\
\cline { 2 - 3 } & 15 & $-281,02$ & $+912,87$ \\
\cline { 2 - 3 } & 25 & $-285,19$ & & $+976,15$ \\
\cline { 2 - 3 } & 37 & $-275,99$ & & $+957,42$ \\
\hline \multirow{3}{*}{ HEPES } & 4 & $-237,18$ & & $+890,71$ \\
\cline { 2 - 3 } & 15 & $-281,80$ & \multirow{2}{*}{$+535,33$} & $+857,71$ \\
\cline { 2 - 3 } & 25 & $-283,92$ & & $+979,82$ \\
\cline { 2 - 3 } & 37 & $-273,94$ & & $+954,08$ \\
\cline { 2 - 3 } & & & & \\
\hline
\end{tabular}

Pela Tabela 3 , nota-se que todos os casos para $\Delta G^{0}$ foram negativos, indicando reação espontânea e, consequentemente, processo favorável de adsorção. Os valores positivos de $\Delta S^{0}$ indicam aumento na desordem total do sistema e o $\Delta \mathrm{H}^{0}$ positivo, por sua vez, sugere a contribuição de interações hidrofóbicas.

A existência de interações de natureza hidrofóbica sugere, a princípio, que deve ocorrer um aumento da capacidade de adsorção e da afinidade com o aumento da temperatura, contudo isso não foi observado. Voltando-se à Tabela 1 e à Tabela 2, nota-se que, nos dois tampões, o aumento de temperatura gerou o resultado esperado ao se passar de $15^{\circ} \mathrm{C}$ a $37^{\circ} \mathrm{C}$, mas que o maior valor ocorreu em $4^{\circ} \mathrm{C}$. Uma possível explicação está na natureza heterogênea 
da adsorção. Havendo essa heterogeneidade, um número maior de moléculas de IgG adsorvidas à matriz pode favorecer o aparecimento de uma segunda camada de adsorção que seria governada por interações hidrofóbicas (proteína-proteína).

\section{CONCLUSÕES}

Foi verificado que a matriz de quitosana/alginato sem corantes imobilizados apresentou resultados significativos de adsorção de IgG humana, tendo afinidade média e, em geral, maiores valores de $\mathrm{Q}_{\mathrm{m}}$ com o tampão HEPES, que teve o maior valor a $4^{\circ} \mathrm{C}$. O modelo de Langmuir-Freundlich apresentou o melhor ajuste para as isotermas dos dois tampões, indicando que há cooperatividade positiva no sistema. Os resultados para o tampão HEPES também se mostraram melhores que aqueles obtidos em matriz com Cibacron Blue F3G4 imobilizado em ensaios de leito fixo. Por fim, verificou-se que o processo de adsorção, no caso, é termodinamicamente favorável e que há a contribuição de interações hidrofóbicas, que podem governar uma segunda camada de adsorção por meio de interações proteína-proteína.

\section{AGRADECIMENTOS}

Os autores agradecem à Fundação de Amparo à Pesquisa do Estado de São Paulo (FAPESP) pelo apoio financeiro recebido para a realização do presente trabalho (processos 2014/23893-3 e 2016/18005-7).

\section{REFERÊNCIAS}

BRADFORD, M.M. A rapid and sensitive method for the quantification of microgram quantities of protein utilizing the principle of protein-dye binding. Anal. Biochem., v. 72, p. 248-254, 1976.

BRESOLIN, I. T. L.; RIBEIRO; M. B.; TAMASHIRO; W. M. S. C.; AUGUSTO; E. F. P.; VIJAYALAKSHMI, M. A.; BUENO, S. M. A. Evaluation of immobilized metal-ion affinity chromatography (IMAC) as a technique for $\mathrm{IgG1}$ monoclonal antibodies purification: the effect of chelating ligand and support. Appl. Biochem. Biotechnol. V. 160, p. 2148-2165, 2010.

CARVAlHO, B. T. C; CONDINO-NETO, A.; SOLE, D.; FILHO, N. R; I Consenso brasileiro sobre o uso de imunoglobulina humana em pacientes com imunodeficiências primárias. Ver. Bras. Alerg. Immunopatol. V. 33, n 3, p. 104-116, 2010.

GONDIM, D.R.; DIAS, N.A.; BRESOLIN, I.T.L.; BAPTISTIOLLI, A.M.; AZEVEDO, D.C.S.; SILVA Jr, I.J. Human IgG Adsorption using Dye-Ligand Epoxy Chitosan/Alginate as Adsorbent: Influence of Buffer System. Adsorption, v. 20, p. $925-$ 934, 2014.

LOWE, C.R., LOWE, A.R., GUPTA, G. New developments in affinity chromatography with potential application in the production of biopharmaceuticals. Journal of Biochemical and Biophysical Methods, v. 49, p. 561-574, 2001.

PRASANNA, R.R., VIJAYALAKSHMI, M.A. Characterization of chelate methacrylate nonolithic disk for purification of polyclonal and noclonal immunoglobulin G. Journal of Chromatography A, v. 1217, p. 3660-3667, 2010. 